\title{
Approximating Probability Densities by Iterated Laplace Approximations
}

\author{
Björn Bornkamp*
}

March 21, 2011

\begin{abstract}
The Laplace approximation is an old, but frequently used method to approximate integrals for Bayesian calculations. In this paper we develop an extension of the Laplace approximation, by applying it iteratively to the residual, i.e., the difference between the current approximation and the true function. The final approximation is thus a linear combination of multivariate normal densities, where the coefficients are chosen to achieve a good fit to the target distribution. We illustrate on real and artificial examples that the proposed procedure is a computationally efficient alternative to current approaches for approximation of multivariate probability densities.
\end{abstract}

keywords: Bayes Factor, Importance Sampling, Markov Chain Monte Carlo, Non-Linear Regression, Normalization Constant

${ }^{*}$ Work done while Björn Bornkamp was Postdoctoral Fellow at Fakultät Statistik, Technische Universität Dortmund, 44221 Dortmund, Germany (email: bornkamp@statistik.tu-dortmund.de). 


\section{Introduction}

Suppose you are given a positive integrable function $\pi(\boldsymbol{x})$ on $\mathbb{R}^{p}$, with unknown normalization constant $Z=\int \pi(\boldsymbol{x}) \mathrm{d} \boldsymbol{x}$ and it is desired to approximate the probability distribution $\pi(\boldsymbol{x}) / Z$. In the context of Bayesian statistics this could for example be the posterior distribution. The idea of the standard Laplace approximation is to maximize $\log (\pi(\boldsymbol{x}))$, resulting in the mode $\tilde{\boldsymbol{x}}$ (i.e. a point with gradient 0 ) and to approximate $\log (\pi(\boldsymbol{x}))$ by a second order Taylor approximation in $\tilde{\boldsymbol{x}}$ (assuming the necessary derivatives of $\log (\pi(\boldsymbol{x}))$ exist). This leads to an approximation of the form

$$
\pi(\boldsymbol{x}) \approx \exp \left(\log (\pi(\tilde{\boldsymbol{x}}))+1 / 2(\boldsymbol{x}-\tilde{\boldsymbol{x}})^{\prime} H(\tilde{\boldsymbol{x}})(\boldsymbol{x}-\tilde{\boldsymbol{x}})\right)
$$

where $H(\tilde{\boldsymbol{x}})=\partial \log \pi(\boldsymbol{x}) / \partial x_{i} \partial x_{j}$ is the Hesse matrix of $\log (\pi(\boldsymbol{x}))$ evaluated at $\tilde{\boldsymbol{x}}$. Essentially, $\pi(\boldsymbol{x})$ is approximated by the kernel of a multivariate normal distribution with mean $\tilde{\boldsymbol{x}}$ and covariance matrix $\tilde{\boldsymbol{\Sigma}}=-H(\tilde{\boldsymbol{x}})^{-1}$. The normalization constant of this approximation is $(2 \pi)^{p / 2}|\tilde{\boldsymbol{\Sigma}}|^{1 / 2} \pi(\tilde{\boldsymbol{x}})$, which itself approximates $Z$. At first sight it might appear simplistic to approximate any distribution by a normal distribution, but in the context of Bayesian statistics this approach is justified by the asymptotic normality of posterior distributions, and often works also for moderate sample sizes; see, for example, Evans \& Swartz (2000, Chapter 4) or Tierney \& Kadane (1986) for more details and DiCiccio, Kass, Raftery \& Wasserman (1997) or Nott, Kohn \& Fielding (2008) for further work in the context of approximating the normalization constant. The recent article by Rue, Martino \& Chopin (2009) successfully uses Laplace approximations for latent variables in latent Gaussian models to approximate

marginal densities, while Haran \& Tierney (2010) use the Laplace approximation to build automatic MCMC algorithms in a related model class.

The Laplace approximation is always unimodal and elliptical. In the case of multiple modes, one can partially overcome this problem by fitting Laplace approximations to each mode (see Gelman, Carlin, Stern \& Rubin (2003, Chapter 12)). Nevertheless, non-elliptical skew posterior distributions remain a challenge and relatively few papers try to improve the Laplace approximation in this regard. One approach is to employ third order derivatives in the Taylor expansion (see for example O'Hagan \& Forster (2004, p. 238-239)). Unfortunately, these can be hard to calculate, particularly in larger dimensions. Due to dominance of the cubic terms, the approximation might also diverge in the tails, so that it might not be integrable. Another approach is to center the normal approximation on the first two moments, instead of mode and negative inverse Hessian at the mode (Minka 2001). Nott, Fielding \& Leonte (2009) consider to improve the Laplace approximation based on numerical integration, in particular for approximating $Z$. 
In this paper the idea of iterating the Laplace approximation is developed. Given an approximation $\tilde{\pi}(\boldsymbol{x})$ of $\pi(\boldsymbol{x})$, the approximation of $\pi(\boldsymbol{x})$ is improved by fitting a Laplace approximation to the residual $r(\boldsymbol{x})=\pi(\boldsymbol{x})-\tilde{\pi}(\boldsymbol{x})$. The current approximation $\tilde{\pi}(\boldsymbol{x})$ fits worst where $r(\boldsymbol{x})$ has its maximum, so that the resulting residual Laplace approximation corrects the deficiencies of fit between $\tilde{\pi}(\boldsymbol{x})$ and $\pi(\boldsymbol{x})$. The new approximation is then given by a linear combination of the starting approximation $\tilde{\pi}(\boldsymbol{x})$ and the residual Laplace approximation. This process is repeated until the approximation does not change considerably. The idea of improving an approximation by fitting the "residuals" of the current approximation and then using a linear combination of the obtained functions, has been applied elsewhere in the statistical literature. It is, for example, at the core of the relaxed greedy algorithm described in Barron, Cohen, Dahmen \& DeVore (2008) (who use dictionaries to model the individual functions) or the boosting technique in machine learning, where this idea is usually called functional gradient descent, see Bühlmann \& Yu (2003). The difference to the procedure presented here is that the Laplace approximation is used to define new elements in the linear combination, rather than relying on a functional basis or dictionaries. Using Laplace approximation has the advantage that it is automatically centered at the point, where the current approximation is worst, i.e., where the residual is largest. In addition, the approximation is a linear combination of multivariate normal densities. This is convenient from a statistical and computational viewpoint (e.g., sampling random variates and evaluation of the density are straightforward).

At the end of the iterative process one hence obtains a global approximation of $\pi(\boldsymbol{x})$, which can be used for subsequent statistical inference directly, or as a proposal distribution for Monte Carlo simulation (such as importance sampling or the independence MetropolisHastings algorithm (Tierney 1994, ch. 2.3)). Building global approximations to obtain proposal distributions has received considerable attention in recent years. An idea appearing repeatedly is that of building a mixture based approximation, while performing the iterative simulation algorithm. In the context of importance sampling this has been considered for example by Cappé, Douc, Guillin, Marin \& Robert (2008) or Ardia, Hoogerheide \& van Dijk (2009). In the context of adaptive MCMC, mixture based approximations have been discussed for example by Andrieu \& Moulines (2006, ch. 7) or Giordani \& Kohn (2010).

We think that iterating Laplace approximations can be a viable alternative to current approaches for building global approximations of $\pi(\boldsymbol{x}) / Z$. In Section 2 this idea will be elaborated in detail, while in Section 3 the methodology will be evaluated on three test examples and a nonlinear regression application. 
(i)

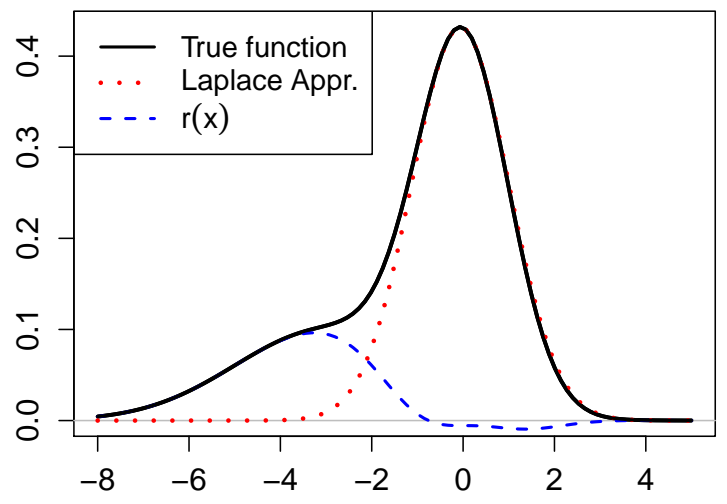

(ii)

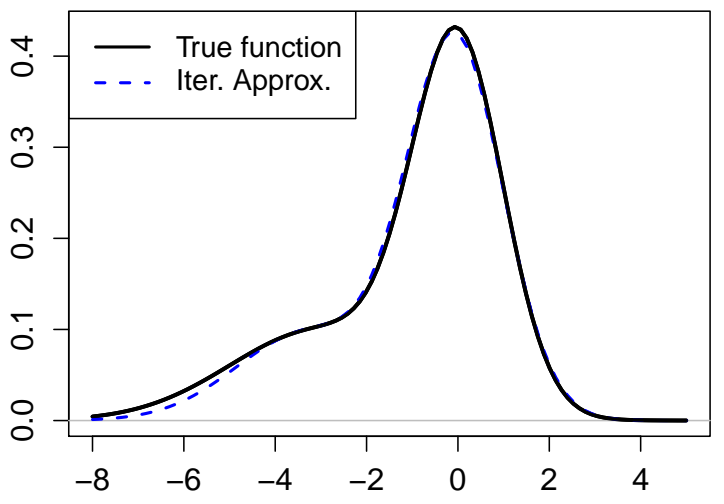

Figure 1: Illustrating the iterated Laplace approximation.

\section{Iterated Laplace Approximations}

The main idea of iterated Laplace approximations, abbreviated iterLap, is to improve the current approximation $\tilde{\pi}(\boldsymbol{x})$ of $\pi(\boldsymbol{x})$ by performing a Laplace approximation of the residual $r(\boldsymbol{x})=\pi(\boldsymbol{x})-\tilde{\pi}(\boldsymbol{x})$, and then taking $w_{1} \tilde{\pi}(\boldsymbol{x})+w_{2} r_{L}(\boldsymbol{x})$ as the new approximation, where $r_{L}(\boldsymbol{x})$ is the (normalized) Laplace approximation of $r(\boldsymbol{x})$ (i.e., a multivariate normal density) and the $w_{j}$ are suitably determined positive coefficients. This procedure is then iterated until a satisfying approximation is achieved. The final approximation of $\pi(\boldsymbol{x})$ will be a linear combination of multivariate normal densities and the normalizing constant of the approximation can be obtained simply by adding the coefficients $w_{j}$. The residual $r(\boldsymbol{x})$ can get negative, which causes a problem when calculating $\log (r(\boldsymbol{x}))$. Hence one should use, for example, the positive function $r(\boldsymbol{x}) 1_{A}(\boldsymbol{x})+\exp (r(\boldsymbol{x})-\tilde{\epsilon}) \tilde{\epsilon} 1_{A^{c}}(\boldsymbol{x})$ as the objective function, where $A=\{x \mid r(\boldsymbol{x}) \geq \tilde{\epsilon}\}$ and $\tilde{\epsilon}$ is a small positive constant. This does not change the residual in the region of interest, where $r(\boldsymbol{x})>0$.

The procedure will be illustrated here by fitting the one-dimensional skew non-normalized density $\pi(x)=\phi(x, 0,1)+0.5 \phi(x,-3,2)$, where $\phi(x, \mu, \sigma)$ denotes the density of the normal distribution with mean $\mu$ and standard deviation $\sigma$. In Figure 1 (i) the true function $\pi(x)$ and the Laplace approximation $\tilde{\pi}(x)$ based on the global maximum are displayed. Due to skewness, the Laplace approximation fits poorly in the left part of $\pi(x)$, where the residual function $r(x)$ consequently has its maximum. Figure 1 (ii) displays the new approximation based on a mixture of the initial Laplace approximation and the Laplace approximation of the residual $r(x)$. The coefficients $w_{1}$ and $w_{2}$ of the two components were chosen to minimize the $L_{2}$ distance between approximation and truth $\pi(x)$. The approximation fits the true function fairly well, despite a remaining small discrepancy in the left tail, which 
could be eliminated by a further iteration of the algorithm.

While the conceptual idea of iterating Laplace approximations is fairly simple, care must be taken when implementing the idea. In the following the computational details of iterated Laplace approximations will be described.

\section{Algorithm}

Iteration 0:

1. Fit a Laplace approximation to each mode of $\pi(\boldsymbol{x})$, to obtain a starting approximation: $\tilde{\pi}_{0}(\boldsymbol{x})=\sum_{j=1}^{J^{(0)}} w_{j} \phi\left(\boldsymbol{x}, \boldsymbol{\mu}_{j}, \boldsymbol{\Sigma}_{j}\right)$, where $\phi(\boldsymbol{x}, \boldsymbol{\mu}, \boldsymbol{\Sigma})$ denotes the density of a multivariate normal distribution with mean $\boldsymbol{\mu}$ and covariance matrix $\boldsymbol{\Sigma}, J^{(0)}$ the number of found modes, $\boldsymbol{\mu}_{j}$ the modes, $\boldsymbol{\Sigma}_{j}$ the negative inverse Hessians of $\log (\pi(\boldsymbol{x}))$ evaluated at the modes and $w_{j}=(2 \pi)^{p / 2}\left|\boldsymbol{\Sigma}_{j}\right|^{1 / 2} \pi\left(\boldsymbol{\mu}_{j}\right)$; see Gelman et al. (2003, Chapter 12) for details on this multiple mode Laplace approximation.

2. Determine for each component in the linear combination a grid of size $n$ that encloses most of its probability mass (see A.1 below for more details). Let $\boldsymbol{X}_{0}$ denote the $n J^{(0)} \times p$ matrix that contains these grid points in the rows.

3. Evaluate $\pi($.$) at \boldsymbol{X}_{0}$, resulting in the vector $\boldsymbol{y}_{0}$ of length $n J^{(0)}$. Also evaluate each of the $J^{(0)}$ component densities in the mixture at $\boldsymbol{X}_{0}$ and write those evaluations in the $n J^{(0)} \times J^{(0)}$ matrix $\boldsymbol{F}_{0}$.

4. Check the stopping criterion (see A.2 below for details); if this is not met initialize $t \leftarrow 1$.

\section{Iteration $t$ :}

1. In this step the residual Laplace approximation is performed to obtain one new mixture component. Select $k$ possible starting values for optimization of the log-residual $\log \left(r(\boldsymbol{x})\right.$ ), with $r(\boldsymbol{x})=\pi(\boldsymbol{x})-\tilde{\pi}_{t}(\boldsymbol{x})$ (see A.3 below for details on selecting starting values). Start a local optimizer at the first starting value, potentially resulting in a maximum $\tilde{\boldsymbol{x}}$ with zero gradient vector. If the Hesse matrix $\tilde{\boldsymbol{H}}$ at $\tilde{\boldsymbol{x}}$ is negative definite, a new mixture component has been found, i.e., one increments the number of components $J^{(t)} \leftarrow J^{(t-1)}+1$, and sets $\boldsymbol{\mu}_{J^{(t)}}=\tilde{\boldsymbol{x}}$ and $\boldsymbol{\Sigma}_{J^{(t)}}=-\tilde{\boldsymbol{H}}^{-1}$. Otherwise, if $\tilde{\boldsymbol{H}}$ is not negative definite one tries the next starting value. If all of the $k$ starting values fail, stop the procedure, as no adequate improvement can be found; otherwise continue to step 2 . 
2. Determine a grid $\boldsymbol{N}_{t}$ of size $n$ for the new component that encloses most of its probability mass (see A.1). Add these points to the current grid $\boldsymbol{X}_{t-1}$ to form $\boldsymbol{X}_{t}=\left(\begin{array}{c}\boldsymbol{X}_{t-1} \\ \boldsymbol{N}_{t}\end{array}\right)$, which is then of size $n J^{(t)} \times p$.

3. Evaluate $\pi($.$) at \boldsymbol{N}_{t}$ and append these evaluations to $\boldsymbol{y}_{t-1}$ to form $\boldsymbol{y}_{t}$. Evaluate all components of the approximation $\tilde{\pi}_{t-1}($.$) at \boldsymbol{N}_{t}$ and the new component at the entire grid $\boldsymbol{X}_{t}$ to form the $n J^{(t)} \times J^{(t)}$ matrix $\boldsymbol{F}_{t}$.

4. Find the coefficients $w_{1}, \ldots, w_{J^{(t)}}$ by minimizing $\left(\boldsymbol{y}_{t}-\boldsymbol{F}_{t}^{\prime} \boldsymbol{w}\right)^{\prime}\left(\boldsymbol{y}_{t}-\boldsymbol{F}_{t}^{\prime} \boldsymbol{w}\right)$ subject to $w_{j} \geq$ 0 for $j=1, \ldots, J^{(t)}$ (see A.4 below for details) and calculate the current approximation of the normalization constant $Z_{t}=\sum_{j=1}^{J^{(t)}} w_{j}$. The current approximation of $\pi(\boldsymbol{x})$ is then $\tilde{\pi}_{t}(\boldsymbol{x})=\sum_{j=1}^{J^{(t)}} w_{j} \phi\left(\boldsymbol{x}, \boldsymbol{\mu}_{j}, \boldsymbol{\Sigma}_{j}\right)$.

5. Check the stopping criteria, if they are not met iterate $t \leftarrow t+1$ (see also A.2).

The points below illustrate computational details in the implementation of the algorithm.

\section{A.1 Grid}

The reason for choosing the grid is to identify regions where $\pi($.$) has positive probability$ mass. In our experience it works well to use a quasi-random sample of the multivariate normal distribution underlying the selected component. For this purpose a randomized quasi-random sample generated by the Sobol sequence is used (as implemented for example in the $\mathrm{R}$ package randtoolbox, Dutang (2009)). Compared to a pseudo random sample this has the advantage that the space is more systematically covered. A default choice of $n$ is discussed below.

\section{A.2 Stopping Criteria}

Different criteria can be used for stopping the iterative process. First one can compare $\boldsymbol{y}_{t}$ and $\tilde{\boldsymbol{y}}_{t}=\boldsymbol{F}_{t}^{\prime} \boldsymbol{w}_{t}$, i.e. stopping the iterative process, when $\max \left|\boldsymbol{y}_{t}-\tilde{\boldsymbol{y}}_{t}\right|<\delta M_{t}$, where $M_{t}=\max _{\boldsymbol{X}_{t}} \pi(\boldsymbol{x})$ and $\delta$ is a small positive number. This criterion assesses the quality of the approximation on the current grid, and stops the process, when there are only small differences between truth and approximation. This criterion is already available at iteration 0 . Another stopping rule is to monitor the normalizing constant $Z_{t}$ of $\tilde{\pi}_{t}(\boldsymbol{x})$. It measures the "volume" of the approximation $\tilde{\pi}_{t}(\boldsymbol{x})$; if $Z_{t}$ does not change further this indicates that the algorithm cannot find more regions, where $\pi(\boldsymbol{x})$ has relevant probability mass. It is not uncommon that two consecutive iterations only lead to small changes in the normalizing constant, so we stop the iterative process, when $Z_{t}$ does not change considerably the third time in a row, i.e., when $\frac{\left|Z_{t}-0.5\left(Z_{t-1}+Z_{t-2}\right)\right|}{Z_{t}}<\epsilon$, where $\epsilon$ is a small positive constant. Note that both of the above stopping criteria (as 
most stopping criteria for iterative calculations), do not guarantee a certain quality of the solution, but with sensible values for $\epsilon$ and $\delta$ (default choices are discussed below) one often obtains a satisfactory result. Note that the procedure can also stop, when no adequate modes can be found in step 1 of iteration $t$, or when a maximal pre-specified number of components $T$ is reached. All of the above criteria will be employed in the examples discussed in the later sections.

\section{A.3 Starting values}

The residual function $r(\boldsymbol{x})$ is often multimodal. Because the used optimizers are designed for finding local optima, it is crucial to use good starting values. In our experience it works well to use starting values where $y_{i} / \tilde{y}_{i}, i=1, \ldots, n J^{(t)}$ is largest; here $y_{i}$ and $\tilde{y}_{i}$ denote the entries of $\boldsymbol{y}_{t}$ and $\tilde{\boldsymbol{y}}_{t}$. This choice works better than, for example, using the values where $y_{i}-\tilde{y}_{i}$ is largest, because the former selects values further apart from the current modes. In our implementation the ten grid values for which the $y_{i} / \tilde{y}_{i}$ is largest are selected, and then clustered with the $k$-means algorithm (Hartigan \& Wong 1979) to obtain $k=3$ starting values. The order in which the starting values are used is determined by the distance to the last added mode, with the values farthest away being tried first. This prevents the algorithm from wandering in only one direction.

\section{A.4 Quadratic Programming}

Solving the constrained least squares problem in step 4 is a quadratic programming problem and can be solved efficiently for example with the algorithm of Goldfarb \& Idnani (1982).

In summary the algorithm needs: The grid size $n$ for each component, the values $\epsilon$ and $\delta$ for the two stopping criteria and the maximum number $T$ of components allowed. Suited default values for those parameters have been determined by experimentation based on a number of example densities, covering a range of different cases observed in practice. A good time-quality trade off for the grid size was obtained for the smallest integer larger than $50 p^{1.25}$. The number 50 has been selected based on the observation that a grid size of 50 is often sufficient in one dimensional cases, the exponent 1.25 has been chosen to obtain a grid size that grows slightly faster than linear in the dimension. For $\delta$ and $\epsilon$ the choices $\delta=0.01$ and $\epsilon=0.005$ were found to work well. The main rationale for these selections is that one should not stop the process before all relevant probability mass has been identified. On the other hand, stopping late will take more time (both for construction of the approximation, as well as for sampling) with only a marginal improvement. For the maximum number of components, $T=20$ was sufficient in the considered examples. All subsequent applications 

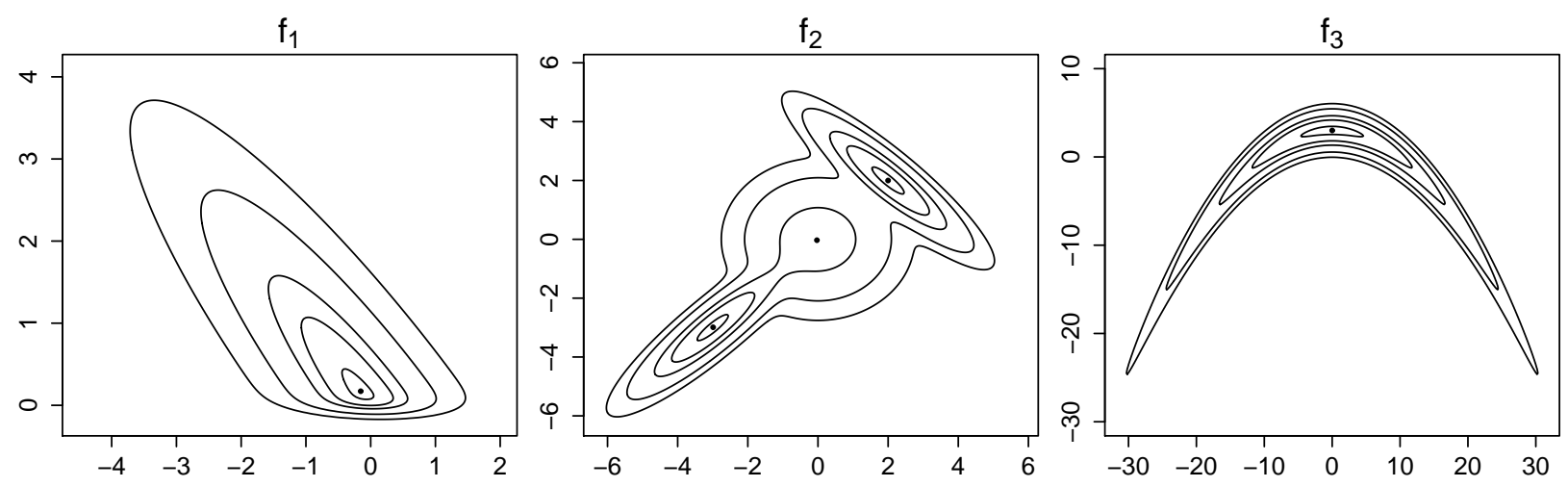

Figure 2: Contour plot for the three test densities. For $f_{3}$ only the two first coordinates are plotted. Black dots $(\bullet)$ denote local maxima and the contour lines are located at 0.9, 0.5, $0.25,0.05$ and 0.01 of the height of the global maximum.

in this paper rely on these default assumptions. When these choices fail, i.e., the algorithm fails to identify parts of the probability mass, one can increase $n$, or decrease $\delta$ and $\epsilon$. Another strategy is to increase the number of starting values at iteration 0 , if complete modes might have been missed at the beginning.

The algorithm was implemented in the $\mathrm{R}$ computing language ( $\mathrm{R}$ Development Core Team 2010). To avoid floating point errors we work on the log-scale whenever possible. When one needs to work on the original scale, for example, in the quadratic programming step or in the optimization of $\log (r(\boldsymbol{x}))$, one can use $\pi^{*}(\boldsymbol{x})=\exp \left(\log (\pi(\boldsymbol{x}))-\log \left(M_{t}\right)\right)$, where $\log \left(M_{t}\right)=\max _{\boldsymbol{X}_{t}} \log (\pi(\boldsymbol{x}))$. In this way excessively small values are avoided.

\section{Applications}

\subsection{Test Cases}

In this section the method is illustrated for three artificial, yet realistic test cases. Common challenges for computational approaches in Bayesian statistics are skew, non-linear and multimodal posterior distributions. These often occur in applications beyond the standard statistical models, for example, when the statistical model is not from an exponential family with conjugate prior and linear predictors. 
The first density $f_{1}$ is a bivariate skew $t$-distribution with 5 degrees of freedom, scale matrix $\boldsymbol{\Omega}=\left(\begin{array}{cc}1 & -0.9 \\ -0.9 & 1\end{array}\right)$, location vector $\boldsymbol{\xi}=(0,0)^{\prime}$ and skewness vector $\boldsymbol{\alpha}=(0,15)^{\prime}$ (see Azzalini \& Capitanio (2003) for details on the parametrization). This density (displayed in Figure 2 left) possesses an extreme non-elliptical skew shape. The second density $f_{2}$ is a mixture of three bivariate normal distributions: $f_{2}(\boldsymbol{x})=0.34 \phi\left(\boldsymbol{x},(0,0)^{\prime}, \boldsymbol{S}_{1}\right)+0.33 \phi\left(\boldsymbol{x},(-3,-3)^{\prime}, \boldsymbol{S}_{2}\right)+$ $0.33 \phi\left(\boldsymbol{x},(2,2)^{\prime}, \boldsymbol{S}_{3}\right)$, with $\boldsymbol{S}_{1}=\left(\begin{array}{ll}1 & 0 \\ 0 & 1\end{array}\right), \boldsymbol{S}_{2}=\left(\begin{array}{cc}1 & 0.9 \\ 0.9 & 1\end{array}\right)$ and $\boldsymbol{S}_{3}=\left(\begin{array}{cc}1 & -0.9 \\ -0.9 & 1\end{array}\right)$ (Gilks, Roberts \& Sahu 1998). This density is multimodal and has a complex local structure, see Figure 2 (middle). The third density is the ten dimensional non-linear banana shaped distribution used in Wraith et al. (2009), with density $f_{3}(\boldsymbol{x}) \propto \phi(t(\boldsymbol{x}), \boldsymbol{m}, \boldsymbol{S})$, where $t(\boldsymbol{x})=\left(x_{1}, x_{2}+\right.$ $\left.b\left(x_{1}^{2}-\sigma_{1}^{2}\right), x_{3}, \ldots, x_{10}\right), \boldsymbol{m}=(0, \ldots, 0)^{\prime}$ and $\boldsymbol{S}=\operatorname{diag}\left(\sigma_{1}^{2}, 1, \ldots, 1\right), b=0.03$ and $\sigma_{1}^{2}=100$ were used, as in Wraith et al. (2009). Figure 2 right, displays the density of the first two components, when the other coordinates are fixed at 0.

iterLap has been applied to these problems with the default tuning parameters. As starting values for the optimization in the initial Laplace approximation in each case one vector consisting only of zeros has been used. For $f_{1}$ iterLap selects 9 components and stops the iterative process, because the algorithm cannot further change the approximation of the normalization constant. For $f_{2}$ the algorithm stops the iterative process after 5 components, because the maximum error on the grid points is achieved. For $f_{3}$ the iterative process is illustrated in some detail in Figure 3, which displays the selected 11 components and the order in which they are selected. The algorithm stops, because the normalization constant of the approximation does not further change considerably. In Figure 3 one can also see that the orientation of the selected components' ellipses fits the underlying local structure of the distribution quite well.

As distance measure to the true density the normalized effective sample size (NESS) has been calculated by an application of importance sampling with the obtained mixture of normal distributions as proposal. NESS lies in $(0,1]$, where larger values correspond to a better fit and NESS is an estimate of a monotonic transformation of the $\chi^{2}$-distance between proposal and true distribution (Kong, Liu \& Wong 1994). It is defined as NESS = $1 /\left(N \sum_{i=1}^{N} \tilde{\omega}_{i}^{2}\right)$, where $N$ is the number of simulated values and $\tilde{\omega}_{i}$ the normalized importance weights. The reported values of NESS in Table 1 are the average over 100 independent runs of importance sampling each with sample size $N=10000$ (standard deviation given in brackets). In addition the means and standard deviations of the marginal distributions have been compared. For this purpose the absolute distance between true and approximated mean and standard deviation were used and divided by the true standard deviation of the corresponding marginal distribution. Deviations from the true values are thus measured in units of the true standard deviation. 


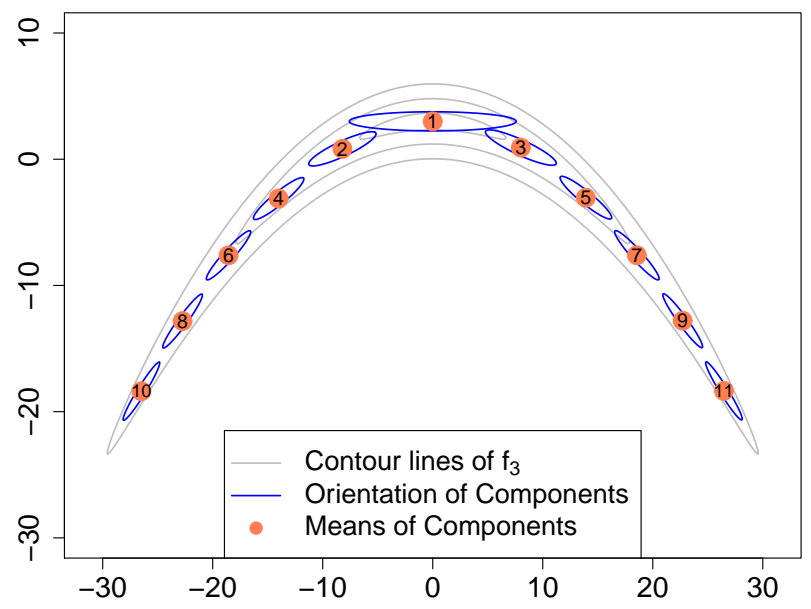

Figure 3: Illustration of iterLap for the first two dimensions of $f_{3}$. The numbers in the dots refer to the order in which components were added.

\begin{tabular}{l|l|ccccc} 
Density & Method & NESS & $\operatorname{Mean}_{x_{1}}$ & $\mathrm{sd}_{x_{1}}$ & $\operatorname{Mean}_{x_{2}}$ & $\mathrm{Sd}_{x_{2}}$ \\
\hline \hline$f_{1}$ & Laplace & $0.04(0.04)$ & 0.72 & 0.49 & 0.88 & 0.59 \\
& iter. Lapl. & $0.65(0.20)$ & 0.02 & 0.16 & 0.05 & 0.11 \\
\hline$f_{2}$ & Laplace & $0.02(0.01)$ & 0.13 & 0.56 & 0.13 & 0.56 \\
& iter. Lapl. & $0.99(<0.01)$ & $<0.01$ & $<0.01$ & $<0.01$ & $<0.01$ \\
\hline \multirow{2}{*}{$f_{3}$} & Laplace & $0.05(0.04)$ & $<0.01$ & $<0.01$ & 0.70 & 0.77 \\
& iter. Lapl. & $0.71(0.04)$ & $<0.01$ & 0.14 & 0.15 & 0.08 \\
\hline
\end{tabular}

Table 1: Normalized Effective Sample Size (NESS) and approximation error in the marginal mean and standard deviation relative to the true standard deviation for the Laplace approximation and the iterated Laplace approximation of $f_{1}, f_{2}$ and $f_{3}$. 
Table 1 displays the results for iterLap and for a standard Laplace approximation (which was used as the starting approximation for iterLap). One can conclude that the iterated Laplace approximation has a substantially better performance than the standard Laplace approximation particularly in terms of NESS, but also for the moments of the marginal distributions.

The results regarding the ten dimensional $f_{3}$ are also quite encouraging: Compared to the results of Wraith et al. (2009, Figure 3), who use adaptive importance sampling based on Cappé et al. (2008), one can observe that a similar median effective sample size was obtained for 10 iterations of adaptive importance sampling. The adaptive importance sampling, however, has a larger variability and needs a total of 100000 function evaluations in each case. Our approach needs a total of around 19000 function evaluations for building the approximation, including evaluations for building the grid as well as evaluations needed for the optimizer and calculating the Hessians. The number of function evaluations is an important machine independent indicator on how fast an algorithm runs, as the other computations needed by the algorithms can usually be neglected, particularly if evaluation of the target distribution is computationally expensive. To get an idea of the actual computation time needed by our $\mathrm{R}$ implementation (which does not exploit that parts of the code can be parallelized): Building the global approximation for $f_{3}$ takes around 2.5 seconds (using a Laptop computer with $1.86 \mathrm{Ghz}$ and 2GB RAM).

\subsection{Nonlinear Regression}

To illustrate iterLap on a real problem, data on monthly averaged atmospheric pressure differences between the Easter Islands and Darwin, Australia over 168 months are used (see Figure 4). These data are taken from the NIST website www.itl.nist.gov/div898/strd/nls/data/enso.shtml. The difference in pressure is of meteorologic importance as it drives the trade winds in the southern hemisphere, and the main purpose of the data analysis is to infer the frequency of periodic cycles. The model for the data is $y_{i} \sim N\left(\mu(i), \sigma^{2}\right)$, where $\mu(i)=$ $\alpha+\sum_{k=1}^{3} A_{k} \sin \left(2 \pi i / \lambda_{k}\right)+B_{k} \cos \left(2 \pi i / \lambda_{k}\right)$ for $i=1, \ldots, 168$. For the conditionally linear parameters $\alpha, A_{k}, B_{k}, k=1,2,3$ independent Cauchy a-priori distributions with median 0 and scale 100 (for $\alpha$ ) and 10 (for $A_{k}, B_{k}, k=1,2,3$ ) were used. For the positive parameter $\lambda_{k}$ independent uniform distributions on $[0,100]$ were employed and for $\sigma$ a gamma distribution with parameters 0.1 and 0.1 . The transformation $\log (\sigma)$ has been used, to obtain a parameter that lies in $\mathbb{R}$. All parameters will be summarized in the vector $\boldsymbol{\theta}$. The likelihood surface is highly multimodal, but there seems to be one dominant mode. As starting value 


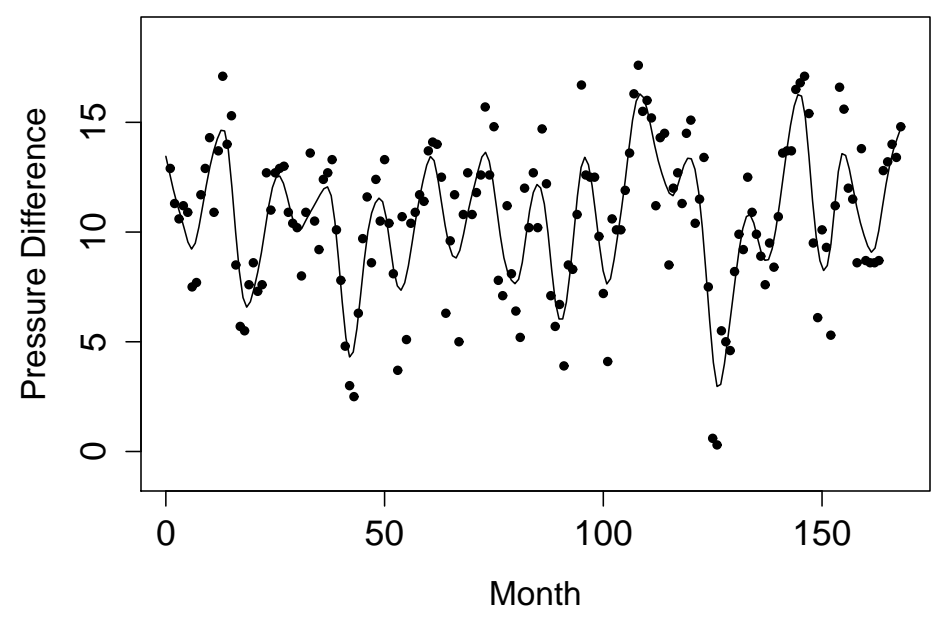

Figure 4: Pressure Difference between the Easter Islands and Darwin, Australia with smoothing spline fit (black line) to give an idea of the conditional mean function.

for the optimization the least squares estimate shown on the NIST website is used (note however that here also $\lambda_{1}$ is treated as unknown, while in their analysis this is fixed to 12).

To compare iterLap to other computational methods, four long MCMC runs were produced (each started at the mode and of size 2500000 with thinning 10 after a burn-in of 10000) and the resulting 1000000 iterations are used as a gold-standard. These runs were obtained with a multivariate random walk Metropolis algorithm implemented in the mcmc package of Geyer (2010), with proposal variance matrix $2.38^{2} / 11 \tilde{\Sigma}$, where $\tilde{\Sigma}$ is the negative inverse Hessian at the mode of the log-density.

Using the default tuning parameters, iterated Laplace approximation selects 12 components and stops the iterative process, because the normalization constant of the approximation does not further improve. For this application the obtained distribution will be used as a proposal distribution for importance sampling. As is common in importance sampling, the Gaussian approximations obtained from iterLap will be replaced by mixtures of t-distributions with equal centering vector and scale matrix and 10 degrees of freedom, because those possess heavier tails. To obtain an unweighted sample, importance sampling resampling with residual resampling was used with sample size 5000 (see Robert \& Casella (2004, Chapter 14) for details on residual resampling).

The iterLap procedure will be compared with two MCMC-based approaches. First the componentwise adaptive random walk Metropolis algorithm is used (see Roberts \& Rosenthal 

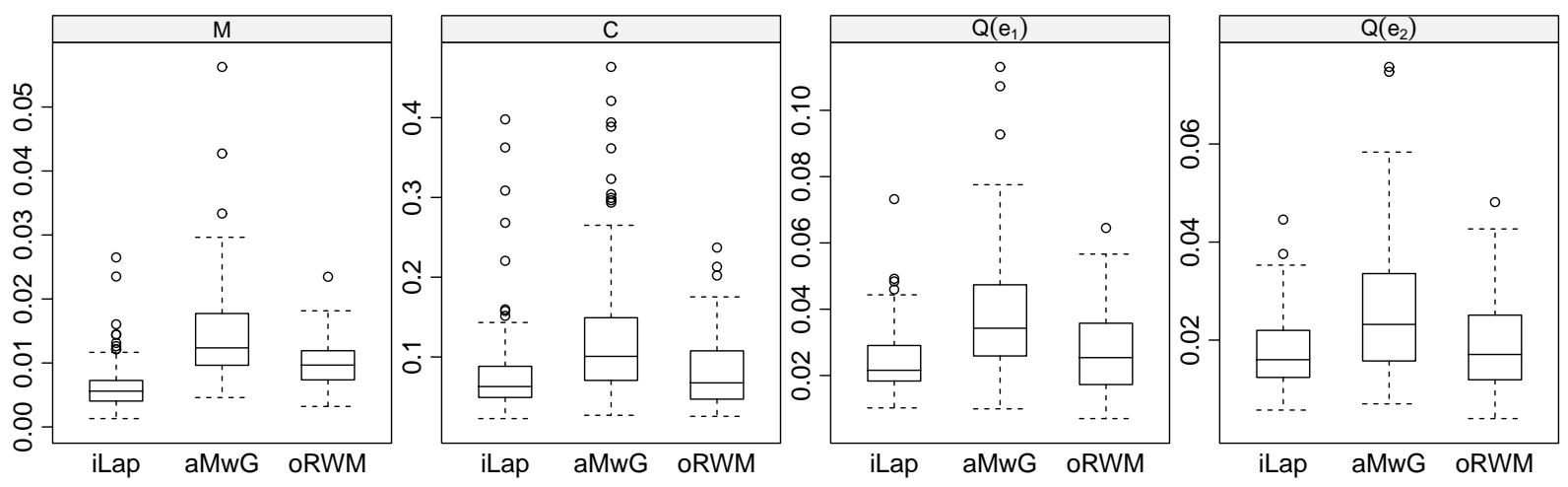

Figure 5: Simulation results for the three methods over 100 repetitions. iLap $\hat{=}$ importance sampling with iterLap, aMwG. $\hat{=}$ adaptive Metropolis within Gibbs, oRWM $\hat{=}$ optimal random walk Metropolis with true covariance matrix.

(2009)). To improve the componentwise updating, the algorithm is applied on the transformed scale $\tilde{\boldsymbol{\theta}}=\tilde{\boldsymbol{\Sigma}}^{1 / 2} \boldsymbol{\theta}+\tilde{\boldsymbol{\mu}}$, with $\tilde{\boldsymbol{\mu}}$ the posterior mode and $\tilde{\boldsymbol{\Sigma}}^{1 / 2}$ the square root of the negative inverse Hessian at the mode calculated from the eigen decomposition. This has the advantage that the covariance matrix is approximately diagonal on the transformed scale. For implementation the function adaptMetropGibbs from the spBayes package (Finley, Banerjee \& Carlin 2010) was used with target acceptance rate set to 0.44 and initial proposal standard deviations set to 2.38. The second MCMC approach is an optimally tuned multivariate random walk Metropolis algorithm. The covariance matrix of the proposal was chosen proportional to the covariance matrix obtained from the gold-standard runs above, with the scaling selected as $2.38^{2} / 11$. This is an algorithm that cannot be used in practice, because the "true" covariance matrix is unavailable a-priori. Nevertheless, this algorithm is of interest as it provides an upper bound on the performance of the adaptive random walk Metropolis algorithm (as described for example in Roberts \& Rosenthal (2009)), which needs to estimate the covariance matrix from its own iterations. In all cases the starting value is chosen equal to the mode of the posterior distribution.

Four performance measures are used to compare the methodologies. First, the estimates of the posterior means are compared by calculating the Mahalanobis distance $M\left(\hat{\boldsymbol{\theta}}, \boldsymbol{\theta}_{\text {true }}\right)=$ $\sqrt{\left(\hat{\boldsymbol{\theta}}-\boldsymbol{\theta}_{\text {true }}\right)^{\prime} \boldsymbol{S}_{\text {true }}^{-1}\left(\hat{\boldsymbol{\theta}}-\boldsymbol{\theta}_{\text {true }}\right)}$, between the empirical average $\hat{\boldsymbol{\theta}}$ from the simulation output and the values $\boldsymbol{\theta}_{\text {true }}$ and $\boldsymbol{S}_{\text {true }}$ obtained from the gold-standard runs. To compare the estimates of the parameter covariance matrix, the spectral norm of the difference between the empirical and true covariance matrices $C\left(\hat{\boldsymbol{S}}, \boldsymbol{S}_{\text {true }}\right)=\sqrt{\lambda_{\max }\left(\boldsymbol{D}^{\prime} \boldsymbol{D}\right)}$ was calculated, where $\boldsymbol{D}=\hat{\boldsymbol{S}}-\boldsymbol{S}_{\text {true }}$ and $\lambda_{\max }($.$) is the function that returns the largest eigenvalue. To$ 
obtain measures of multivariate goodness of fit, the quantiles $q_{0.05}^{(j)}, q_{0.1}^{(j)}, q_{0.15}^{(j)}, \ldots, q_{0.95}^{(j)}$ along the first two eigenvectors $e_{j}, j=1,2$ of the true correlation matrix were calculated from the gold-standard runs and then compared to the quantiles obtained from the simulations $Q\left(e_{j}\right)=\frac{1}{19} \sum_{i \in\{0.05,0.1, \ldots, 0.95\}}\left|\hat{q}_{i}^{(j)}-q_{i}^{(j)}\right|$, for $j=1,2$.

All three algorithms were applied to the problem and repeated 100 times. The iterLap procedure requires around 28000 function evaluations values in total (both for building the approximation and the 5000 evaluations for importance sampling). For the MCMC based algorithms twice as many function evaluations namely a total of 60000 iterations were allowed, with 10000 burn-in and thinning rate 10, so that in summary also 5000 iterations are obtained. From the results displayed in Figure 5 one can conclude that importance sampling with iterLap works very well compared to the other approaches in all performance measures (particularly for the posterior moments), although it uses fewer function evaluations. In addition it is easy to obtain a reliable estimate of the normalizing constant via importance sampling, while this is more complicated to obtain reliably from MCMC output.

Returning to the original aim of the data analysis, the resulting posterior means for the frequency parameters $\left(\lambda_{1}, \lambda_{2}, \lambda_{3}\right)$ are given by $(11.9,44.1,26.8)^{\prime}$ months with standard deviations $(0.04,1.1,0.36)^{\prime}$. These frequencies can be traced to the yearly cycle $\left(\lambda_{1}\right)$, El Niño $\left(\lambda_{2}\right)$ and the Southern Oscillation $\left(\lambda_{3}\right)$, see the NIST website for details.

\section{Discussion}

Compared to traditional function approximation or regression function estimation, globally approximating a positive integrable function $\pi(\boldsymbol{x})$ proportional to a probability density is a considerably more difficult problem. The main complication is that it is a-priori unclear where to approximate $\pi(\boldsymbol{x})$, i.e., where most of the probability mass of $\pi(\boldsymbol{x})$ is located. In this article the iterated Laplace approximation has been introduced to solve this twofold problem of identification of regions with relevant probability mass, and approximation of $\pi(\boldsymbol{x})$ in these regions. The methodology starts with a simple Laplace approximation, and then iteratively applies Laplace approximation to the residual between truth and current approximation, until a stopping criterion is satisfied. By optimizing the residual in each step of the procedure, the algorithm identifies regions with relevant probability mass, where the current approximation fits poorly and an improvement is needed. Once a mode and the local curvature is determined, the new component is added to the approximation, and the 
coefficients are determined by minimizing the $L_{2}$ distance between truth and approximation on a grid.

In this paper the methodology has been evaluated in three test cases and one real example with positive results. In the case of the ten dimensional banana shaped example, the approach obtained similar results as adaptive importance sampling based on a mixture of $t$ distributions with improved computational efficiency in terms of function evaluations. Further, in the real data example, where two state of the art MCMC algorithms were applied, the iterated Laplace approximation showed a very competitive performance with a smaller number of function evaluations.

As for all analytical approximations, it is difficult to assess the quality of the obtained iterated Laplace approximation in a concrete modelling situation. Hence, its main value is to use it as a proposal distribution for Monte Carlo techniques. These techniques allow to assess the quality of the approximation and correct for deficiencies of fit by rejecting or weighting samples. While the focus in this paper has been on importance sampling techniques (where the effective sample size can be used to assess the quality of the approximation), one can, of course, also use the approximation in the context of MCMC techniques that employ a global proposal distribution, such as the independence Metropolis-Hastings algorithm or the rejection sampling Metropolis-Hastings algorithm (Tierney 1994, ch. 2.3), in these cases reliable MCMC standard errors can be calculated to evaluate the quality of the simulation (see Flegal, Haran \& Jones (2008)).

A primary application of the methodology might be non-linear models as applied in diverse fields, for example early phase clinical trials or cosmology. In these models the posterior distribution can be skew and multimodal, and one usually cannot design Gibbs moves to directly sample from the full conditional distributions. Nevertheless, the algorithm was also tested with success on a variety of other applications, for example dose-response estimation, Gaussian process regression and simple hierarchical models.

In higher dimensional problems it gets difficult to build a global approximation of non-trivial posterior distributions, and the proposed methodology is no exception: In these cases local MCMC moves often become more efficient, although the iterated Laplace approximation typically still provides an improvement over the standard Laplace approximation in terms of building a global approximation of the posterior and approximating the normalization constant. A computational concern with regard to the methodology is the need for numerically calculating the Hessian matrices. Depending on the problem, this might become unstable (for example when the objective function is flat in the neighborhood of the mode, or the mode lies on a ridge) and, in larger dimensions, computationally expensive. A partial so- 
lution, as suggested by a referee, is to use a structured form of the covariance matrix (e.g., a diagonal matrix). This reduces the computational burden in high dimensional cases and stabilizes computations. The downside would be that a worse fit is obtained by the added components and it is likely that more mixture components are required to obtain an adequate approximation. Another challenge for the iterLap methodology are situations when the target density contains a large number of strongly separated modes. A partial solution in these cases is to use more widely dispersed starting values for the starting approximation (at iteration 0). Alternatively, one could also consider to use tempered version of the residual function to avoid getting trapped in minor local modes.

\section{Acknowledgements}

The author would like thank Frank Bretz and David Ohlssen for proofreading the manuscript, and the Editor, Associate Editor and two referees for helpful comments and suggestions that improved the presentation of this article.

\section{References}

Andrieu, C. \& Moulines, É. (2006) On the ergodicity properties of some adaptive MCMC algorithms, The Annals of Applied Probability 16, 1462-1505.

Ardia, D., Hoogerheide, L. F. \& van Dijk, H. K. (2009) Adaptive mixture of Student$\mathrm{t}$ distribution as a flexible candidate distribution for efficient simulation: The $\mathrm{R}$ package 'AdMit', Journal of Statistical Software 29, 1-32.

Azzalini, A. \& Capitanio, A. T. (2003) Distributions generated by perturbation of symmetry with emphasis on a multivariate skew $\mathrm{t}$ distribution, Journal of the Royal Statistical Society B 65, 367-389.

Barron, A. R., Cohen, A., Dahmen, W. \& DeVore, R. A. (2008) Approximation and learning by greedy algorithms, Annals of Statistics 36, 64-94.

Bühlmann, P. \& Yu, B. (2003) Boosting with the $\mathrm{L}_{2}$ loss: Regression and classification, Journal of the American Statistical Association 98, 324-339.

Cappé, O., Douc, R., Guillin, A., Marin, J.-M. \& Robert, C. P. (2008) Adaptive importance sampling in general mixture classes, Statistics and Computing 18, 447-459. 
DiCiccio, T. J., Kass, R. E., Raftery, A. \& Wasserman, L. (1997) Computing Bayes factors by combining simulation and asymptotic approximations, Journal of the American Statistical Association 92, 903-915.

Dutang, C. (2009) randtoolbox: Generating and Testing Random Numbers, R package version 1.07 .

Evans, M. \& Swartz, T. (2000) Approximating Integrals via Monte Carlo and Deterministic Methods, Oxford University Press, Oxford.

Finley, A. O., Banerjee, S. \& Carlin, B. P. (2010) spBayes: Univariate and Multivariate Spatial Modeling, $\mathrm{R}$ package version 0.1-8.

Flegal, J., Haran, M. \& Jones, G. (2008) Markov chain Monte Carlo: Can we trust the third significant figure, Statistical Science 23, 250-260.

Gelman, A., Carlin, J. B., Stern, H. S. \& Rubin, D. B. (2003) Bayesian Data Analysis, 2nd edition, Chapman and Hall.

GeYer, C. J. (2010) mcmc: Markov Chain Monte Carlo, R package version 0.7-5.

Gilks, W. R., Roberts, G. O. \& Sahu, S. K. (1998) Adaptive Markov chain Monte Carlo through regeneration, Journal of the American Statistical Association 93, 10451054.

Giordani, P. \& Kohn, R. (2010) Adaptive independent Metropolis-Hastings by fast estimation of mixtures of normals, Journal of Computational and Graphical Statistics 19, $243-259$.

Goldfarb, D. \& Idnani, A. (1982) Dual and primal-dual methods for solving strictly convex quadratic programs, in J. Hennart (ed.), Numerical Analysis, Springer Verlag, Berlin, pp. 226-239.

Haran, M. \& Tierney, L. (2010) On automating Markov chain Monte Carlo for a class of spatial models, Technical report, Department of Statistics, Pennsylvania State University, USA.

Hartigan, J. A. \& Wong, M. A. (1979) A k-means clustering algorithm, Applied Statistics 28, 100-108.

Kong, A., Liu, J. S. \& Wong, W. H. (1994) Sequential imputation and Bayesian missing data problems, Journal of the American Statistical Association 89, 278-288. 
MinkA, T. (2001) Expectation propagation for approximate Bayesian inference, in J. S. Breese \& D. Koller (eds.), UAI '01: Proceedings of the 17th Conference in Uncertainty in Artificial Intelligence, pp. 362-369.

Nott, D. J., Fielding, M. \& Leonte, D. (2009) On a generalization of the Laplace approximation, Statistics and Probability Letters 79, 1397-1403.

Nott, D. J., Kohn, R. J. \& Fielding, M. (2008) Approximating the marginal likelihood using copula.

*http://arxiv.org/abs/0810.5474

O'Hagan, A. \& Forster, J. (2004) Kendall's Advanced Theory of Statistics, Volume 2B: Bayesian Inference, 2nd edition, Arnold, London.

R Development Core Team (2010) R: A Language and Environment for Statistical Computing, R Foundation for Statistical Computing, Vienna, Austria, ISBN 3-900051$07-0$.

Robert, C. P. \& Casella, G. (2004) Monte Carlo Statistical Methods, 2nd edition, Springer, New York.

Roberts, G. O. \& Rosenthal, J. S. (2009) Examples of adaptive MCMC, Journal of Computational and Graphical Statistics 18, 349-367.

Rue, H., Martino, S. \& Chopin, N. (2009) Approximate Bayesian inference for latent Gaussian models by using integrated nested Laplace approximations, Journal of the Royal Statistical Society B 71, 319-392.

Tierney, L. (1994) Markov chains for exploring posterior distributions, Annals of Statistics 22, 1701-1762.

Tierney, L. \& Kadane, J. (1986) Accurate approximations for posterior moments and marginal densities, Journal of the American Statistical Association 81, 82-86.

Wraith, D., Kilbinger, M., Benabed, K., Cappé, O., Cardoso, J.-F., Fort, G., Prunet, S. \& Robert, C. P. (2009) Estimation of cosmological parameters using adaptive importance sampling, Physical Review D 80, 023507. 